IS HEART RATE VARIABILITY A VALUABLE METHOD TO INVESTIGATE CARDIAC AUTONOMIC DYSFUNCTION IN SUBJECTS WITH LEUKEMIA? A SYSTEMATIC REVIEW TO EVALUATE ITS IMPORTANCE IN CLINICAL PRACTICE

Heart Rate Variability and leukemia

Jociele M. Kirizawa ${ }^{1}$, David M. Garner ${ }^{2}$, Claudia $\mathrm{Arab}^{3}$, Vitor E. Valenti ${ }^{1}$

${ }^{1}$ Autonomic Nervous System Center, Sao Paulo State University, UNESP, Presidente Prudente, SP, Brazil. ${ }^{2}$ Cardiorespiratory Research Group, Department of Biological and Medical Sciences, Faculty of Health and Life Sciences, Oxford Brookes University, Headington Campus, Gipsy Lane, Oxford, OX3 0BP, United Kingdom. ${ }^{3}$ Department of Medicine (Cardiology), Federal University of São Paulo (UNIFESP), Sao Paulo, SP, Brazil.

* Correspondence to: Vitor E. Valenti

UNESP, Av. Hygino Muzzi Filho, 737. Mirante

17.525-900 - Marília, SP. Brazil

Phone: +5514 3402-1300

E-mail: vitor.valenti@unesp.br 


\begin{abstract}
Impaired cardiovascular and autonomic function during treatment and during recovery from leukemia has been indicated. In this context, heart rate variability (HRV) is a non-invasive measure that describes the oscillations of the intervals between consecutive heart beats (RR intervals); influenced by the autonomic nervous system. We intend to review literature showing HRV changes in leukemia subjects. The articles selected in the current review were attained up to March 2018, and the search was limited to articles in English language, published in peer-reviewed journals, with both adult and child age samples. The articles were investigated in the five electronic databases: PubMed, Physiotherapy Evidence Database PEDro), Cochrane Clinical Trials, Scientific Electronic Library Online - SciELO and Excerpta Medica dataBASE - EMBASE. Towards the end of the research, 9 studies were included. Subjects undergoing treatment for leukemia have reduced HRV, signifying decreased vagal control of heart rate. The subjects that undertook leukemia treatment and their survivors experienced a reduction in HRV with subsequent recovery, but the recovery time is ill defined. HRV is reduced in leukemia subjects who progress to neuropathy secondary to chemotherapy, accompanied by cardiac dysfunction. We advocate the use of HRV to evaluate autonomic function and decide the treatment to prevent autonomic impairment in leukemia subjects.
\end{abstract}

Keywords: Autonomic Nervous System; Cardiovascular Physiology; Heart Rate; Leukemia, Myeloid Acute, Lymphoid Acute 


\section{MAIN HEADINGS}

This review indicates appropriate studies that assessed HRV in subjects with leukemia. The references demonstrated reduced HRV in leukemia subjects who progressed to neuropathy secondary to chemotherapy, accompanied by cardiac dysfunction. Thus, HRV can be applied to evaluate autonomic dysfunction and possibly help to modify the treatment with the purpose of preventing further autonomic function damage. 


\section{INTRODUCTION}

Lymphoid leukemia is the principal neoplasm affecting children and adolescents worldwide. Its incidence is $25 \%$ to $30 \%$, and has two age peaks. The first is between three and seven years old and the second is over forty years old. Risk factors are related to age and responses to chemotherapy, yet, the identification of recurrent genetic alterations has facilitated the refinement of the individual prognosis and clinical management [1-3].

Leukemia is a malignant and progressive hematological disease that originates in the bone marrow where the blood cells are produced. It is noticeable by the proliferation and mutations of these cells, and may or may not occupy other locations, invading the circulatory system, since it is a blood neoplasm. It is classified as an acute $(95 \%)$ or chronic $(5 \%)$ leukemia [2].

Acute myeloid leukemia and acute lymphoblastic leukemia are amongst the acute leukemias and are highly aggressive, necessitating early treatment. Chronic leukemias, including chronic myelogenous leukemia and chronic lymphocytic leukemia are categorized by proliferation of mature cells. In this grouping of leukemia, the mutation permits the maintenance of differentiation capacity and cell maturation, with an increase in the number of mature cells in the bone marrow and the peripheral blood. The disease progression is slow; yet, it is followed by an accelerated phase and may later develop into acute leukemia. The most prevalent type, in children, is lymphoblastic leukemia. The main clinical manifestations are fever, bleeding, anemia, fatigue and recurrent infections [2, 4]. Furthermore, the literature reports impaired cardiovascular and autonomic function in leukemia during treatment and improvement after the necessary pharmacological treatment [5-7].

In this way, heart rate variability (HRV) is a simple and non-invasive method that describes the oscillations of the intervals between consecutive heart beats (RR intervals), and is influenced by the autonomic nervous system. Its applicability is vast and is able to identify phenomena related to autonomic function in healthy individuals, athletes and subjects with diseases $[8,9]$.

There appears to be an immunological interaction between the malignancy and the peripheral nervous system dysfunction, suggesting that the HRV of subjects with leukemia is more exaggerated in those with a more compromised clinical feature [6]. So, autonomic function is affected in cancer subjects. This could result from the cancer or cancer treatments [10].

A cohort study evaluated 651 subjects with hematologic malignancies submitted to autonomic cardiac evaluation at the cancer outpatient clinic between 2008 and 2011. This study proposed an association between HRV (specifically in the time domain) and subjects' survival time [11].

HRV analysis delivers valued information for subjects under physiological and pathological conditions [10]. Given that the interaction between cancer, chemotherapy and HRV that has been previously defined, we raised the following questions: what is the evidence for the effects of leukemia on HRV? Is there a connection between the leukemia treatments and HRV deviations? Is HRV a good way to evaluate subjects with leukemia? To answer these questions, we performed a systematic review that investigated the use of HRV in leukemia subjects. 


\section{METHOD}

We performed a systematic review according to the 'Preferred Reporting Items for Systematic Reviews and Meta-Analyses' (PRISMA) Statement [12].

\section{Information sources}

The articles in the current review were obtained in journals up to March 2018. These searches were limited to articles in English language, published in peer-reviewed journals, and with adult and child samples only. The articles were investigated in five electronic databases: PubMed (https://www.ncbi.nlm.nih.gov/pubmed), Physiotherapy Evidence Database - PEDro (https://www.pedro.org.au/), Cochrane Clinical Trials (https://www.cochranelibrary.com), Scientific Electronic Library Online - SciELO (http://www.scielo.org) and Excerpta Medica dataBASE - EMBASE (https://www.embase.com).

\section{Search strategy, inclusion and exclusion criteria}

The following terms were applied for the combination of groups of descriptors: "autonomic nervous system", "heart rate variability", "sympathetic nervous system", "vagal nerve", "parasympathetic nervous system", "leukemia" and "humans". The Boolean operator "AND” was necessary in the grouping of the synonyms of the descriptors of the same cohort.

These searches were undertaken without a limit on the year of publication. No filters were necessary to select the studies.

Initially, we commenced with a selection of titles related to the topic. This selection was based on evidence that related HRV with leukemia; duplications were excluded. Moreover, manuscripts that examined leukemia and varied types of cancers. Next, abstracts were assessed, according to those that studied HRV in subjects with leukemia only. We excluded abstracts unrelated to that issue. The full texts were examined, then those that were not suitable regarding the exclusion criteria were excluded in the current review. Likewise, a search was made in the references of the full texts.

So as to increase reliability in the selection of articles, all search and selection stages were reviewed independently by two investigators, and after reading the articles, reached a consensus to establish which articles should be included [12].

\section{Data extraction process}

Data extraction and management of the involved articles were achieved in duplicate (two reviewers independently). We nominated articles based on the PICOS (Subject, Intervention, Control, Outcomes, Study Type) criteria:

\section{Study eligibility criteria}

\section{Subject}

We included studies with humans who were being treated or have undergone leukemia and other types of cancer treatment.

\section{Intervention / Control}

We included references that involved studies evaluating HRV in leukemia subjects and survivors, with or without a control group. We also involved studies that evaluated the effects of pharmacological and non-pharmacological interventions on HRV. 


\section{Outcomes}

The principal outcome measured were the linear and nonlinear indices of HRV: RMSSD (square root of the mean square of the differences between adjacent normal RR intervals), SDNN (standard deviation of all normal RR intervals), pNN50 (percentage of adjacent RR intervals with duration difference greater than 50 milliseconds), HF (high frequency), LF (low frequency), LF/HF ratio (Relation between low and high frequency components), SD1 (dispersion of points perpendicular to the identity line, instantaneous record of beat-to-beat variability), SD2 (scatter points along the identity line, longterm record).

\section{Study Type}

The references selected included any type of study design, yet, we did not include letters to editor, commentaries and reviews.

\section{Data analysis}

The leading and essential criterion was that all details concerning HRV analysis should be described in selected studies, explicitly: description of protocol, data collection, instruments and HRV analysis methods. Next, datasets were evaluated based on the subjects, ethical considerations (informed written consent and committee ethic approval), and detailed data collection procedures in selected studies. At least two of the following items were required to be considered for the current review: (a) inclusion and exclusion criteria, (b) comparison between groups, (c) sample loss, and (d) sample size.

We followed guiding principles from the Grades of Recommendation, Assessment, Development, and Evaluation (GRADE) Working Group [13]. The main feature to be considered in the strength of evidence was study design, categorized as observational studies (low evidence) and randomized trials (high evidence). Study quality (detailed study methods and execution) and presence of limitations were considered in strength of evidence analysis.

\section{RESULTS}

\section{Selection of studies}

In the preliminary literature search, we identified 161 publications in databases. After eliminating the duplicates, 159 studies had their titles and abstracts read. After the first stage, 13 articles needed to be read in entirety. At the end of the research, nine studies were included. The reviewers approved the identification of the remaining nine studies using the abovementioned eligibility criteria. Figure 1.

\section{Characteristics of studies.}

The specific characteristics of each study were detailed in Table 1 with the following information: Authors' name, year; Population; HRV analysis and Main Results. The revised studies addressed the following topics: Four manuscripts that evaluated the effects of chemotherapy on autonomic modulation assessed through HRV, with at least two data collections; three manuscripts that investigated HRV in leukemia survivors (post-treatment); and two manuscripts that examined the effects of different treatments on HRV in leukemia subjects.

\section{Chemotherapy \& HRV}

In this evaluation, four articles addressed the effect of chemotherapy on autonomic modulation assessed through HRV: two evaluated the effect of treatment with 'VinCristine', - a chemotherapy medication $[5,14]$. One study evaluated the effect of arsenic trioxide in a group that was unsuccessful 
with traditional chemotherapy treatment [15]. Another single investigation evaluated the effect of hematopoietic stem cell transplantation after treatment with high doses of chemotherapy [16].

In all studies, HRV was revealed to be reduced in leukemia [5, 14-16]. Also, three studies described that HRV tended to increase gradually with discontinuation of treatment, yet, recovery time is ill defined and could take months to years $[5,14,15]$.

In one study the subjects presented cardiac arrhythmia and QT prolongation, perhaps as a consequence of damage in cardiac autonomic regulation [15].

\section{Post-Treatment \& HRV}

In this appraisal, three articles evaluated HRV in leukemia treatment survivors. Only one of the reviewed study's had a healthy control group [7]. The results strengthened the concept that the treatment largely affects the parasympathetic system. Nevertheless, LF activation in the orthostatic position is infrequently noticed and the small decrease in HR advocates that there is an alteration in the sympathetic system too. The authors grouped the population by gender and compared the control to the leukemia group. The results indicated that the HR autonomic control of young and healthy women is together more complex and susceptible to damage caused by neurotoxic substances, such as chemotherapy. Furthermore, this study reinforced the hypothesis that cardiac neural regulation assessed by HRV is transformed in subjects who have their leukemia treated, but not reversed after the end of their treatment [9].

A study conducted by Zeller et al, [17] evaluated adult survivors of childhood leukemia treatment and split them into two groups: the first group included subjects suffering from chronic fatigue, and the control group included subjects who did not suffer chronic fatigue. Head-up tilt test was completed for cardiovascular and autonomic analysis. The subjects' remained in both positions for five minutes, lying flat and at an inclined slope of $20^{\circ}$ for Electrocardiogram (ECG) analysis. There was no alteration in the results between the groups. This may be justified by the time or slopes applied that were possibly insufficient to generate sufficient cardiovascular and autonomic changes.

One more study has established through HRV, that is decreased in subjects with leukemia, and this highlighted that there is a sympathetic predominance in relation to the parasympathetic system [6].

\section{Alternative Therapies \& HRV}

In this study, two articles addressed HRV and alternative treatments [18, 19]. Kemper et al, in order to evaluate complementary relaxation treatments, directed two studies [18, 19]. The first study applied energy healing-based therapies as a tool to assess the relaxation state of children with leukemia [18]. Two evaluations were achieved and the subjects were their own controls. Also, the technique was performed by a person unfamiliar to the children. There was inferior sympathetic activation when the subjects were exposed to energy healing-based therapy. In contrast, there was no significant effect on the equivalent scale of stress. Since it was a study with a large range of ages, and in some cases, the parents of subjects assisted completion of the stress scale. Additionally, the study did not report how parents completed the scale and the hypothesis raised was that they projected their own feelings or did not have the sensitivity to subtle changes in the children's temperaments. Previously, it was suggested that the sensation of well-being is related to the increase of the activity of the parasympathetic nervous system and decrease of the activity of the sympathetic nervous system [18]. 
In a further study, Kemper et al, [19] performed two assessments to analyze the impact of music on the relaxation feeling in the same population. In that study, the stress scale presented relaxant and well-being effects of music, whilst during HRV a reduction in the parasympathetic control of the HR was reported.

Yet again, parents completed the stress scale with the help of their children and reported high relaxation and low stress on the first questionnaire. It is worth highlighting that relaxation was the sole item with expressive results.

An unexpected finding was the reduction in the parasympathetic regulation of the cardiac rhythm, represented by the HF band [19].

\section{DISCUSSION}

In order to provide a suitable analysis regarding the use of HRV in leukemia, we performed a systematic review. In general, the analysis of selected studies indicated that: 1) subjects undergoing treatment for leukemia have reduced HRV, suggesting decreased vagal control of HR; 2) subjects who underwent leukemia treatment and survived have a reduction in HRV with subsequent recovery, but the recovery time is still ill defined.

Although the first article of this review is from 1989, 'VinCristine' is a medication that is still currently a part of the standard leukemia treatment protocol and, the authors were able to form a small and homogeneous sample with children newly diagnosed with leukemia. All children in the study began treatment with six doses of 'VinCristine'. This pharmacological treatment was shown to weaken the interaction between breathing and autonomic HR control, which suggests a parasympathetic damage in leukemia children. In the aforementioned study, HRV in children with leukemia significantly decreased during 'VinCristine' treatment. Yet, this reduction appears to be alterable with the cessation of the medication [14].

Recently, another study evaluated the effect of 'VinCristine' on neuropathy focusing on autonomic dysfunction in children with leukemia [5]. Although peripheral neuropathy was more prevalent than autonomic neuropathy when associated with 'VinCristine' use, the two conditions seemed to occur concomitantly in that study. The deviations appear caused by this drugs regression at the end of its use, yet, the recovery time is not well defined, and could take months to years. This study selected only subjects who presented severe neuropathy previously detected through the medical diagnostic plan and did not account at which stage of treatment the subjects were at [5]. Together, these datasets suggest that the clinical team should be careful concerning the use of 'VinCristine', since it is shown to impair autonomic function.

In contrast, Kamath et al, demonstrated that the impairment of the autonomic HR control induced by antineoplastic agents is not reversible in survivors who received leukemia treatment including radiotherapy [7].

An article published by Nevruz et al, reinforced that HRV is decreased in subjects with leukemia. This study was founded on biochemical analysis; the leukemia subjects had low hemoglobin and hematocrit, reflecting an anemic state. So, they suggested undertaking research with a group composed of anemic subjects to exclude the possibility of influence of anemia on HRV reduction caused by leukemia. 
This article confirmed an autonomic imbalance in subjects with leukemia and provided substantial evidence concerning prediction of subclinical neuropathy in subjects with leukemia [6].

Considering the numerous changes that leukemia induces physiologically, chronic fatigue is one of the complaints. Zeller et al, reported a higher score in the fatigue questionnaire in leukemia subjects compared to control subjects. Although the leukemia group had lower blood acetylcholine levels, which led us to expect diminished HRV; there was no significant difference of HRV between groups. In the head-up tilt test, the groups presented the expected changes, hence: increased blood pressure and HR. Conceivably, if the authors applied other tests or protocols with the treadmill stress test the results could be different [17].

Since leukemia is a serious disease and requires rapid treatment initiation, some studies $[15,16]$ have inevitably revealed some bias. In the Poreba study (2014), the subjects were submitted to high doses of chemotherapy followed by transplantation of hematopoietic stem cells. That study demonstrated significantly decreased HRV following receiving hematopoietic stem cell transplantation when compared prior to receiving chemotherapy [16].

The same study described changes in HRV and heart rate turbulence, which were indicated to be related to the transplantation procedure [16]. Yet, it is acknowledged that chemotherapy can cause a decrease in HRV [20]. So as to elucidate this query, an additional evaluation would be necessary at the end of the chemotherapy, nonetheless the subjects were debilitated and at risk of death and thus this additional evaluation is not possible.

A similar situation was investigated by Takeshita et al, the study evaluated HRV in subjects who had failed chemotherapy treatment and initiated daily therapy with arsenic trioxide. Overall, all subjects presented reduced HRV, cardiac arrhythmias and QT prolongation, undoubtedly due to damage in cardiac autonomic regulation. The parameters examined in the first evaluation were performed three weeks after treatment onset due to urgency and risk of death [15].

The results presented by Kemper et al, although significant in the analysis of HRV, when compared to the analogue stress scale seem contradictory. It is worth stating that this study highlighted the difficulty of working with children. The authors elected for a reduced stress scale (considering the additional time that parents and children would remain in the outpatients' clinic), which may have prejudiced the results. Some children, owing to age, needed parental help to fill the scale by raising the hypothesis of parents projecting their own feelings and/or not perceiving subtle changes in children's temperaments $[18,19]$.

Alternative treatments were also revealed in this systematic review. Music was one of the therapies included [19]. In this study, the authors described music as a variable able to relax the body, reducing anxiety, fatigue and stress and increasing vitality. The decrease in the parasympathetic regulation of the heart rhythm, represented by a weakened HF band; can be justified by the fact that it is not pleasant for children to remain 30 to 40 minutes at rest, listening to unfamiliar music. Considering well-being and relaxation, in order to familiarize the children with the procedures, if more than one visit was required and, if they played music the children liked, they could become more relaxed [19].

Another alternative therapy used was energy healing-based therapy [18]. The authors evaluated the impact of this intervention on HRV and stress in subjects with leukemia. As a key result, it was unable to 
find significant influence of energy healing-based therapy on the aforesaid variables. So, we should be vigilant with alternative therapies for cancer treatment [18].

Our review offers some significant points to be highlighted. 1) Most of the studies were composed only by volunteers with diagnoses of leukemia, which implied a small statistical sample. 2) Regarding the references cited, we found an array of studies with larger samples, diagnosis of different types of cancers and age ranges. 3) There were no randomized clinical trials and meta-analysis. So, this systematic review did not contain high evidence studies.

\section{CONCLUSIONS}

In summary, we reported suitable studies that assessed HRV in subjects with leukemia. The references demonstrated HRV reduction in leukemia subjects who progressed to neuropathy secondary to chemotherapy, accompanied by cardiac dysfunction. So, HRV can be enforced to evaluate autonomic impairment and, if at all possible, change the clinical treatment to prevent further impairment of autonomic function. The relevance of employing HRV as an indicator of risk factor for evaluating and identifying health impairments related to autonomic function is encouraging. We emphasize HRV as a clinically reliable and complementary approach to evaluate early diagnosis of autonomic dysfunction in leukemia. The use of HRV is proposed to lower impairment of autonomic function in those subjects with leukemia.

\section{Acknowledgments}

Dr. Vitor E. Valenti receives financial support from the National Council for Scientific and Technological Development (CNPq, Brazil) and Foundation of Support for Research from Sao Paulo state (FAPESP).

\section{Compliance with ethical standards}

\section{Conflict of interest}

The authors declare that they have no conflict of interest.

\section{REFERENCES}

1. Terwilliger T, Abdul-Hay M (2017) Acute lymphoblastic leukemia: a comprehensive review and 2017 update. Blood Cancer J 7:e577. https://doi.org/10.1038/bcj.2017.53

2. Pokharel M (2012) Leukemia : A Review Article

3. Terreri MTRA, Hilário MOE, Nakamura C, et al (2012) Musculoskeletal manifestations as the onset of acute leukemias in childhood. J Pediatr (Rio J) 78:481-4. https://doi.org/10.2223/jped.903

4. De Kouchkovsky I, Abdul-Hay M (2016) 'Acute myeloid leukemia: a comprehensive review and 2016 update.’ Blood Cancer J 6:e441-e441. https://doi.org/10.1038/bcj.2016.50 
5. Nazir HF, AlFutaisi A, Zacharia M, et al (2017) Vincristine-induced neuropathy in pediatric patients with acute lymphoblastic leukemia in Oman: Frequent autonomic and more severe cranial nerve involvement. Pediatr Blood Cancer 64:e26677. https://doi.org/10.1002/pbc.26677

6. Nevruz O, Yokusoglu M, Uzun M, et al (2007) Cardiac autonomic functions are altered in patients with acute leukemia, assessed by heart rate variability. Tohoku J Exp Med 211:121-6. https://doi.org/10.1620/tjem.211.121

7. Kamath M V, Halton J, Harvey A, et al (1998) Cardiac autonomic dysfunction in survivors of acute lymphoblastic leukemia in childhood. Int J Oncol 12:635-40.

https://doi.org/10.3892/ijo.12.3.635

8. ChuDuc H, NguyenPhan K, NguyenViet D (2013) A Review of Heart Rate Variability and its Applications. APCBEE Procedia 7:80-85. https://doi.org/10.1016/j.apcbee.2013.08.016

9. Acharya UR, Joseph KP, Kannathal N, et al (2006) Heart rate variability: A review. Med. Biol. Eng. Comput. 44:1031-1051

10. Guo Y, Palmer JL, Strasser F, et al (2013) Heart rate variability as a measure of autonomic dysfunction in men with advanced cancer. Eur J Cancer Care (Engl) 22:612-616. https://doi.org/10.1111/ecc.12066

11. Guo Y, Koshy S, Hui D, et al (2015) Prognostic Value of Heart Rate Variability in Patients With Cancer. J Clin Neurophysiol 32:516-520. https://doi.org/10.1097/WNP.0000000000000210

12. Liberati A, Altman DG, Tetzlaff J, et al (2009) The PRISMA statement for reporting systematic reviews and meta-analyses of studies that evaluate health care interventions: explanation and elaboration. J Clin Epidemiol 62:e1-e34. https://doi.org/10.1016/j.jclinepi.2009.06.006

13. Atkins D, Eccles M, Flottorp S, et al (2004) Systems for grading the quality of evidence and the strength of recommendations I: Critical appraisal of existing approaches The GRADE Working Group. BMC Health Serv Res 4:38. https://doi.org/10.1186/1472-6963-4-38

14. Hirvonen HE, Salmi TT, Heinonen E, et al (1989) Vincristine treatment of acute lymphoblastic leukemia induces transient autonomic cardioneuropathy. Cancer 64:801-5. https://doi.org/10.1002/1097-0142(19890815)64:4<801::AID-CNCR2820640406>3.0.CO;2-E

15. Takeshita A, Uehara A, Shinjo K, et al (2004) Impairment of heart rate variability control during arsenic trioxide treatment for acute promyelocytic leukemia. Leukemia 18:647-648. https://doi.org/10.1038/sj.leu.2403248 
16. Poręba M, Poręba R, Gać P, et al (2014) Heart Rate Variability and Heart Rate Turbulence in Patients with Hematologic Malignancies Subjected to High-Dose Chemotherapy in the Course of Hematopoietic Stem Cell Transplantation. Ann Noninvasive Electrocardiol 19:157-165. https://doi.org/10.1111/anec.12108

17. Zeller B, Ruud E, Havard Loge J, et al (2014) Chronic fatigue in adult survivors of childhood cancer: associated symptoms, neuroendocrine markers, and autonomic cardiovascular responses. Psychosomatics 55:621-9. https://doi.org/10.1016/j.psym.2013.12.005

18. Kemper KJ, Fletcher NB, Hamilton CA, McLean TW (2009) Impact of healing touch on pediatric oncology outpatients: pilot study. J Soc Integr Oncol 7:12-8. https://doi.org/10.2310/7200.2009.0005

19. Kemper KJ, Hamilton CA, Mclean TW, Lovato J (2013) NIH Public Access. 64:105-109. https://doi.org/10.1203/PDR.0b013e318174e6fb.Impact

20. Coumbe BGT, Groarke JD (2018) Cardiovascular Autonomic Dysfunction in Patients with Cancer. Curr Cardiol Rep 20:69. https://doi.org/10.1007/s11886-018-1010-y 
Figure 1. Search process results according PRISMA flow diagram

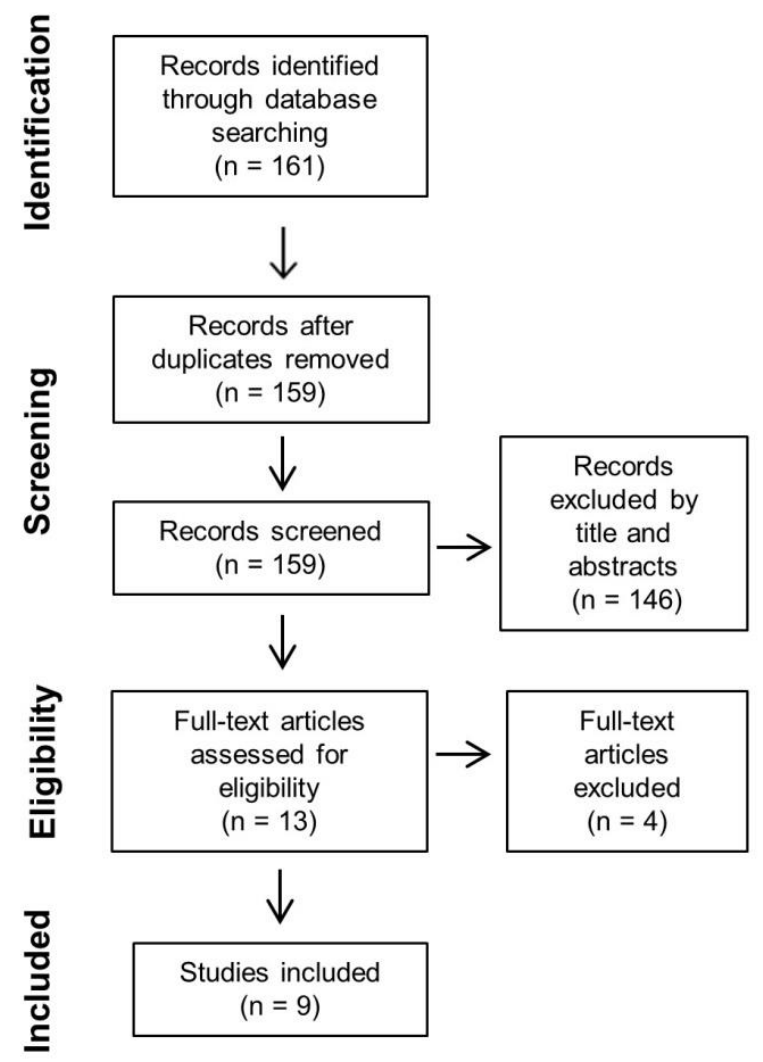


Table I: Summary of selected studies investigating the clinical importance of HRV analysis in leukemia patients.

\begin{tabular}{llll}
\hline Selected articles & Study participants & HRV analysis & Main Results \\
\hline Hirvonen et al. 1989 & Acute lymphoblastic & HRV was evaluated through & HRV was reduced during treatment with \\
& $\begin{array}{l}\text { leukemia treated with } \\
\text { chemotherapy. }\end{array}$ & ECG. Two tests were carried & subsequent recovery after treatment. \\
& out, one was performed in the & \\
(five men, four & first dose of Vincristine. \\
& women) & The second was held between \\
& Age range: $2.5-14$ & the fifth and sixth dose of \\
& Vincristine. \\
& It was performed a follow-up \\
& from 3 $3^{\text {rd }}$ to 22 $2^{\text {nd }}$ week. \\
& Protocol: 5 minutes \\
& spontaneous breathing, \\
& followed by 2 minutes of deep \\
& and controlled breathing.
\end{tabular}

Kamath et al. 1998

Leukemia Group: $\mathrm{n}$ n =

34 (13 women).

Age range: $5-21$ years

old.

Five subjects treated

with cranial radiation,

seven with fractional

minutes in Orthostatism. hours Holter.

As a familiarization, the
Bench press: LH/HF and LF were higher subjects went to the laboratory and ECG was recorded 30 minutes in bench press and 10 cranial radiation and 21 and HF decreased in the Leukemia Group compared to the control group $(\mathrm{p}<0.001)$. Orthostatism: HR, LH/HF, and LF were higher and HF lower in the control group. No difference in HRV time domain was noted. 
with conventional

radiotherapy.

Control Group: 34

subjects (15 women).

Takeshita et al. 2004 Six subjects with acute

promyelocytic

leukemia who relapsed

after treatment with

chemotherapy.

Age Range:

Uninformed

Nevruz et al. 2007

Intervention group: 36

subjects with acute

hours Holter.

leukemia recently

diagnosed. (14 acute

lymphoblastic

leukemia, 22 acute

myeloblastic leukemia)

(11 women). treatment. in the control group.

Genre: Bench Press, LF/HF was decreased in women than men (both groups).

Control: LF difference throughout the day among women.

Leukemia: Attenuated HF in women.

Cranial radiation effect: Significant

differences between patients who received traditional radiation and those who received hyper-fractional radiation.

Higher values of LF/HF and LF were observed in the conventional radiation group compared to control group.

Daily treatment with arsenic

QT wave prolongation appeared in all trioxide (minimum of 25 days subjects $(\mathrm{p}<0.04)$

and a maximum of 60 days).

HRV significantly decreased during HRV was evaluated through treatment: $\mathrm{LF}(\mathrm{p}=0.028)$, SDNNA

24-hour Holter after three $(p=0.043), \operatorname{RMSSD}(p=0.042)$ and $\mathrm{pNN} 50$ weeks of treatment and one $(p=0.024)$.

week prior to termination of

HRV was analyzed via 24 HRV was decreased in patients with leukemia: SDNN, SDANN, pNN50 $(p<0.05)$. 
Mean Age $34 \pm 16$

years old.

Control group: 32

healthy subjects.

(9 women).

Mean Age: $30 \pm 10$

years old.

Kemper et al. 2008

47 subjects ( 9 women), $\quad$ Cohort study

In the subjective stress scale, the participants

Kemper et al. 2009

Zeller et al. 2014
36 leukemia subjects

and 11 subjects with

another type of cancer.

Mean age: $10.4+5.1$

years old.

Age Range: 8 to $17.9 \quad$ Score ranged from $0-10$.

years old.

Five subjects with

leukemia and one with

another type of cancer

(three men).

Mean age: $8.7 \pm 4.3$

years old.

They were in

maintenance or

consolidation

treatment.

conducted between May 2004 and May 2007.

Subjects responded to a stress scale with help from their

parents.

HRV was analyzed.

Cohort study.

There were 2 visits.

Visit 1: usual care and rest in quiet room for 20 minutes.

Visit 2: usual care and rest in quiet room, receiving energy healing-based therapy. At both visits, the children answered a stress questionnaire before and after the procedure.

HRV was analyzed.

Intervention group: $\mathrm{n}=$ Subjects performed the Head32 (25 women) up tilt test: they were placed gave a high score for the positive points and a low score on the negative points on the first visit. Only the relaxation item showed a significant result after the intervention with the music (HF, $\mathrm{p}<0.01)$.

There was a significant decrease in the negative state of the stress scale after the energy healing based therapy. Total power was decreased during healing touch therapy $(\mathrm{p}<0.05)$ 


\begin{tabular}{|c|c|c|c|}
\hline & survivors of leukemia & horizontally on the orthostatic & Autonomic Symptoms also improved: \\
\hline & treatment. & table and remained for five & $\downarrow$ Palpitations $(\mathrm{p}<0.001), \downarrow$ feeling \\
\hline & Mean age: 32.7 years & minutes recording the $\mathrm{RR}$ & intermittently heat and cold $(\mathrm{p}<0.004)$, \\
\hline & old. & intervals and additional five & $\downarrow$ lightheadedness $(\mathrm{p}<0.02), \downarrow$ watery diarrhea \\
\hline & Age range: 22.7 to 50.7 & minutes with a slope of 20 & $(\mathrm{p}<0.001)$ in the control group. \\
\hline & years old. & degrees. & $\uparrow$ Acetylcholine $(\mathrm{p}<0.002)$ in the control \\
\hline & Control: & Blood pressure and HRV were & group. \\
\hline & $\mathrm{n}=52(26$ women $)$ & analyzed. & $\downarrow$ Urine norepinephrine $(\mathrm{p}<0.017)$ in control \\
\hline & subjects. & Subjects also answered fatigue & group. \\
\hline & Mean age: 34 years & questionnaire. & There was no significant difference between \\
\hline & old. & Blood and urine analysis & the groups regarding HRV. \\
\hline & Age range: 20.5 to 53.1 & (Epinephrine, cortisol, & \\
\hline & years old. & norepinephrine) were made. & \\
\hline Poreba 2014 & 38 subjects with & First examination: Before & Individuals with hematopoietic malignancies \\
\hline & leukemia, 18 with & chemotherapy treatment, & treated with hematopoietic stem cell \\
\hline & leukemia lymphoid and & followed by cell & transplantation evidenced decreased HRV \\
\hline & myeloid, seven with & transplantation. & and HR turbulence after stem cells \\
\hline & Hodgkin's lymphoma, & 22 patients received & administration and chemotherapy. \\
\hline & seven with multiple & autologous cells and 16 & \\
\hline & myeloma. & patients received allogenic & \\
\hline & Patients with & cells & \\
\hline & hematological & Blood tests. & \\
\hline & malignancies & ECG and Holter-24 hours & \\
\hline & submitted to high doses & were performed for HRV & \\
\hline & of chemotherapy, in a & analysis. & \\
\hline & course of & Second examination: About & \\
\hline & hematopoietic stem cell & 20 days after completing the & \\
\hline & transplantation & cell transplantation, followed & \\
\hline & $\mathrm{n}=15 \mathrm{~F}, \mathrm{n}=23 \mathrm{M}$ & by chemotherapy. Holter- 24 & \\
\hline & Mean age: $42.88 \pm$ & hours was repeated for HRV & \\
\hline
\end{tabular}


13.49 .

Nazir et al. 2017 analysis.

19 subjects selected

Head-up tilt test: they were

through the electronic

medical record from

placed on the orthostatic table

for five minutes and then

2006 to 2016 (10 men).

Survivors who received

Vincristine as

chemotherapeutic and

evolved with

neuropathy.

Age Range: 2 to 14

years old. positioned on a 70 -degree

inclination, remaining in that

position for 10 minutes.

The following cardiovascular

parameters were assessed:

Heart rate, blood pressure,

stroke volume, peripheral

resistance.

HRV was analyzed in the time

domain.
The authors concluded that HRV was able to provide significant clinical information regarding autonomic dysfunction.

Legend: HRV: heart rate variability; ECG: electrocardiogram; SDNN: standard deviation of all normalto-normal intervals; LF: low frequency; HF: high frequency; RMSSD: square root of the mean of the sum of the squares of differences between successive normal-to-normal intervals; HRV index: number of all normal-to-normal intervals divided by the maximum of all normal-to-normal intervals; LF/HF ratio: low to high frequency ratio VLF: very low frequency; SDANN: standard deviation of the averages of normalto-normal intervals in all 5 min periods of the recording; PNN50: number of pairs of successive normalto-normal intervals differing by more than $50 \mathrm{~ms}$, divided by the total number of normal-to-normal intervals; TP: total power; ms: milliseconds; $\mathrm{ms}^{2}$ : milliseconds squared; nu: normalized units; 
